\title{
Evaluating Thermal Corrections for Adsorption Processes at the Metal/Gas Interface
}

Romain Réocreux, ${ }^{\dagger}$ Carine Michel, ${ }^{\dagger}$ Paul Fleurat-Lessard ${ }^{\ddagger}$ Philippe Sautet, $,{ }^{\mathbf{f}}, \S$ and Stephan N. Steinmann*,†

$\dagger$ †niv Lyon, Ecole Normale Supérieure de Lyon, CNRS Université Lyon 1, Laboratoire de Chimie UMR 5182, 46 allée d'Italie, F-69364, LYON, France

$\ddagger$ Université de Bourgogne Franche-Comté(UBFC), Institut de Chimie Moléculaire de l'Université de Bourgogne (ICMUB), UMR CNRS 6302, 9 avenue Alain Savary 21078 Dijon,

France

IDepartment of Chemical and Biomolecular Engineering, University of California, Los Angeles, Los Angeles, CA 90095, USA

$\S$ Department of Chemistry and Biochemistry, University of California, Los Angeles, Los Angeles, CA 90095, USA

E-mail: stephan.steinmann@ens-lyon.fr

\begin{abstract}
Adsorption and desorption steps are key for active catalysts and rely on a subtle balance between enthalpic and entropic terms. While the enthalpic term is becoming ever more accurate through density functional development, the entropic term remains underrated and its precise determination a great challenge. In this work, we have performed extensive first principles thermodynamic integration (TI) simulations for the
\end{abstract}


adsorption of small (e.g., CO) to larger (e.g., phenol) molecules at metallic surfaces and compared their adsorption free energies to the values obtained by vertical, static statistical mechanics approximations to thermal corrections invoking three different approximations for the low-frequency modes. We have found an excellent agreement between the vertical corrections and the TI for minima, for both weakly bound systems (e.g. $\mathrm{CO}_{2}$ and formic acid) and strongly chemisorbed molecules such as phenol or CO. While the treatment of the low-frequency modes has a minor impact on the agreement with TI, all vertical corrections systematically overestimate activation energies by $0.1-0.2$ eV compared to TI, demonstrating a noticeable lowering of activation barriers. As a result of this study, we suggest that the vertical corrections and in particular the standard harmonic approximation can be safely applied to chemisorption minima, while the activation energies are likely to be overestimated. Hence, if a greater accuracy than $\sim 0.2 \mathrm{eV}$ is required for activation free energies, we recommend to use thermodynamic integration, which for small to medium-sized molecules in gas phase is accessible with a reasonable computational effort, but requires a dense sampling in the transition state region.

\section{Introduction}

In heterogeneous catalysis, the reaction mechanism can be decomposed into three main steps: adsorption of the reactants onto the surface, bond rearrangements on the surface from the reactants to the products and desorption of the products to the gas or liquid phase. ${ }^{1}$ Desorption is the mirror process of adsorption and both are governed by the delicate balance between enthalpy and entropy. Although the adsorption of a molecule is usually accompanied with an endothermic deformation thereof, the adsorbate/surface interaction generally predominates, making the overall adsorption process exothermic. ${ }^{2}$ However, the loss of translational and rotational entropy upon adsorption increasingly counterbalances the exothermicity with higher temperature. Therefore, increasing the temperature reduces 
the adsorption free energy and thus potentially leads to lower surface coverage. Lower coverages are equivalent to lower effective concentrations of starting material but go along with facilitated release of products. Hence, reaching the best compromise between entropy and enthalpy is key for active and selective catalysts. ${ }^{3,4}$ Similarly, for the self-assembled monolayer (SAM) formation, the enthalpy/entropy balance is very subtle and can lead to phase transitions as a function of the temperature and pressure. ${ }^{5}$ To facilitate the design of heterogeneous catalysts and the understanding of SAM formation, accessing the enthalpy and entropy of adsorption is thus critical.

Determining the energy of adsorption of small molecules at $0 \mathrm{~K}$ at the solid/gas interface by density functional theory (DFT) has become standard over the last twenty years. ${ }^{6,7}$ Although functional development remains an active field of research, ${ }^{8-12}$ conceptually and operationally, the procedure to determine these energies is clear. On the experimental side, the adsorption enthalpies can be precisely measured by micro-calorimetry, ${ }^{13,14}$ which serves as a benchmark for the functional development. The same cannot be said for adsorption free energies, where the entropy of the adsorbed state eludes precise determination, both from the experimental and theoretical point of view. Also in surface science the interest in determining the entropy has surged. ${ }^{15}$ For instance, extracting thermodynamical data from temperature programmed desorption (TPD) heavily relies on assumptions regarding the entropy or, equivalently, on the pre-exponential factors of the rates of desorption processes. ${ }^{16,17}$ For several decades, the lack of understanding had led most surface science groups to assume a pre-exponential factor of $10^{13} \mathrm{~s}^{-1}$ when analyzing TPD data. Only recently Campbell and co-workers have proposed a way to estimate desorption entropies and hence pre-exponential factor in a more thorough and accurate fashion. ${ }^{15}$

Several groups working in computational heterogeneous catalysis and surface science have started investigating in detail the passage from $0 \mathrm{~K}$ to free energies at finite temperature. ${ }^{18,19}$ In computational chemistry, the most rigorous approach is to map out the potential energy surface at the DFT level and then solve the associated Schrödinger equation for the nuclei. ${ }^{20}$ 
However, this is only practical for mono-atomic adsorbates. In homogeneous catalysis ${ }^{21,22}$ and for biologically relevant adsorbates on metallic, ${ }^{23}$ calcite, ${ }^{24}$ clay ${ }^{25}$ or oxide ${ }^{26,27}$ surfaces in water, thermodynamic integration (TI), ideally based on ab initio molecular dynamics (AIMD), is considered reliable for determining free energy differences. TI is almost exclusively based on classical Newtonian mechanics (i.e., no tunneling) and does not take into account the quantized nature of the motion of nuclei. Albeit important for low temperature reactions involving bonds with very light atoms (hydrogen mostly), these quantum effects ${ }^{28}$ are negligible for desorption compared to the inaccuracies due to the evaluation of the energy (force field or DFT). Extracting the changes of entropy from molecular dynamics either requires very long simulations to achieve reasonable statistical uncertainties or several simulations at different temperatures. ${ }^{29}$ Alternatively, an approximate thermodynamic post-treatment allows the retrieval of entropy, at least in liquids. ${ }^{30}$

Currently, most studies in heterogeneous catalysis simply apply statistical mechanical formulae to the minima and transition states (TS) found on the potential energy surface at 0 K to compute the corresponding free energies. Applying the lattice-gas approximation (loss of translational and rotational degrees of freedom upon adsorption) and harmonic vibrational degrees of freedom is computationally the most efficient way to evaluate the entropy of the adsorbed species. If necessary, configurational entropy can be taken into account at a similar negligible cost. Nonetheless, for weakly bound adsorbates that have low diffusion barriers on the surface, the lattice-gas approximation is expected to be inaccurate. Therefore, the 2D ideal gas (free 2D translation) approximation has been advocated. ${ }^{31}$ In recent years, Campbell and co-workers have developed interpolations between the lattice-gas and the 2D ideal gas in order to smoothly switch from one to the other as a function of temperature. ${ }^{15,32,33}$ Similarly, rotational degrees of freedom on the surface are usually lost, but for weekly adsorbed molecules the rotation around the surface normal can be considered active. Low frequency modes, which contribute most to vibrational entropy, are usually poorly described within the harmonic approximation and therefore benefit from ad hoc approximations, ${ }^{18}$ anharmonic 
treatments, ${ }^{34,35}$ analogies to hindered rotations ${ }^{36}$ or cut-offs. ${ }^{37}$ For the remaining of the manuscript, these different flavors of approximations will be referred to under the general designation vertical thermal corrections.

In this contribution we compare, for the first time, vertical thermal corrections to TI based on AIMD for the metal/gas interface for a variety of adsorbates, spanning the range from weakly to strongly adsorbed species. To the best of our knowledge, such a direct comparison has has only been performed for systems that are heavily investigated for advanced entropy treatments in heterogeneous catalysis, i.e., zeolites, ${ }^{38,39}$ where the effect of anharmonic corrections, ${ }^{34,35}$ modified vertical thermal corrections ${ }^{40}$ and AIMD $^{41-43}$ have mostly been discussed separately.

The comparison between TI and the vertical thermal corrections requires care in the definition of the reference state (for which $\mathrm{E}$ or $\mathrm{F}$ is zero). In vertical thermal corrections, the reference state is very clearly defined with analytical formula. In TI, however, the equivalent can be difficult to converge. ${ }^{44,45}$ Hence, while standard states are routinely used in statistical mechanics, the straightforward reference is often used in TI, i.e. without readjusting for the available volume/surface. ${ }^{46}$ This practice has been criticized $^{47}$ since the absence of a unique reference makes comparisons between systems problematic. Note that the double decoupling method by McCammon and co-workers is designed to speed up convergence allowing for a proper definition of the reference state, but relies on an additional constraining potential, which we have not applied herein. ${ }^{44}$ As discussed in the SI, we herein use the middle between the two faces of the slab within our periodic setup, the so-called "desorption transition state", as the reference for the TI. Here we show that this kind of AIMD simulation has now become feasible for standard systems studied in metal based heterogeneous catalysis. Although they remain computationally expensive, they allow assessing the accuracy of the vertical thermal corrections. 


\section{Computational Details}

All computations have been performed within the VASP code. ${ }^{48,49}$ The wave function was converged to $10^{-6} \mathrm{eV}$, while using the automatic optimization of the real-space projectors, normal precision settings and a $400 \mathrm{eV}$ cut-off basis set in combination with the PAW formalism for treating the electron-ion interaction. ${ }^{50,51}$ All $0 \mathrm{~K}$ geometries have been relaxed until the forces acting were smaller than $0.02 \mathrm{eV} / \AA$. All fully optimized geometries can be found in the SI. For $\mathrm{CO}_{2}$ and $\mathrm{HCOOH}$ in a $\mathrm{p}(3 \times 3)$ unit cell, the PBE functional ${ }^{52}$ is applied, while for $\mathrm{C}_{6} \mathrm{Cl}_{6}$ and phenol the optPBE-vdW approach ${ }^{8}$ is chosen to treat a $\mathrm{p}(4 \mathrm{x} 4)$ cell. The Brillouin zone is integrated with a 3x3x1 Monkhorst-Pack K-point mesh. Three metallic layers are considered with the bottom one fixed in the bulk position. Four-layer slab have been considered for phenol and $\mathrm{C}_{6} \mathrm{Cl}_{6}$ but have not showed significant deviations for the vertical corrections as compared to three-layer slabs (see Table S3 in the SI), even though the overall adsorption energy at $0 \mathrm{~K}$ is lowered. The vacuum size is set to about $16 \AA$, so that the position $8 \AA$ above the surface corresponds to the middle of the box and represents the point furthest away accessible in our computations.

For $\mathrm{CO}$ on $\mathrm{Ru}(0001)$, we adopted a setup in close correspondence to ref 53 , i.e., a $\mathrm{p}(2 \times 2)$ unit cell of 4 layers, with a Brillouin sampling of $5 \times 5 \times 1$ and the BEEF-vdW density functional. We found a significant contribution $(\sim 0.09 \mathrm{eV})$ of the ZPE only for the adsorption of $\mathrm{CO}$ and did, therefore, not include ZPE in any of our comparisons to be consistent between TI and the vertical corrections.

In the vertical thermal corrections, translations in the gas-phase are treated according to the Sackur-Tetrode equation, keeping only the in-plane translational degrees of freedom to compare with our TI profiles that are referenced to the desorption transition state, which is defined as a 2D gas (see SI). The rotational modes of gas phase molecules are treated within the rigid rotor approximation. In combination with the harmonic treatment of all vibrational modes, this corresponds to the widely used Rigid Rotor and Harmonic Oscillator (RRHO) approach. Low frequency vibrational modes can, however, be particularly anharmonic. This is 
of great concern as these modes dominate the vibrational entropy. We have tested two different flavors for dealing with the low frequency modes (see Table S1). In the renormalization approach all the low-frequencies are replaced by the renormalization value itself $\left(100 \mathrm{~cm}^{-1}\right)$ to ensure the consistency of the number of modes from one structure to another. ${ }^{54}$ The cut-off approach is a pragmatic solution which consists of ignoring them below a cut-off value. ${ }^{37}$ The cut-off of $50 \mathrm{~cm}^{-1}$ proposed in ref 37 was found to give negligible changes with respect to RRHO. In agreement with the cut-off chosen for the renormalization approach, we have set it here to $100 \mathrm{~cm}^{-1}$, a value that modifies RRHO more severely. By doing so, the number of vibrational modes taken into account may vary from one structure to another.

Molecular dynamic simulations were performed in the NVT ensemble. All hydrogen atoms were treated as tritium (mass $=3 \mathrm{amu}$ ) in order to use a time step of $1 \mathrm{fs}$. Since the nuclei are treated classically in AIMD, ZPE is not retrieved in AIMD. The use of tritium reduces the changes in ZPE compared to hydrogen. For a fair comparison with the vertical thermal corrections, tritium was also used in the static calculations where the ZPE was not included (see Table S2). The use of tritium also modifies the anharmonicity of the O-H stretch vibrations. ${ }^{55}$ In view of the statistical uncertainties from the TI, the slight bias towards the validity of RRHO is not critical for the conclusions of this study. Two Andersen thermostats were used to control the temperature $(300 \mathrm{~K})$ of the metal surface and the molecule individually. In the Andersen thermostat, the velocities of particles are randomly re-initialized from the Maxwell-Boltzmann distribution with a given probability. This probability was set to $1 \%$ per atom and time step. ${ }^{56}$ The use of a stochastic thermostat achieves a homogeneous energy distribution among the different degrees of freedoms in gas-phase simulations. ${ }^{21} \mathrm{~A}$ comparison with a more sophisticated, but global, Lowe-Anderson thermostat ${ }^{57}$ showed good agreement for the few points tested. Note that the CSVR thermostat, ${ }^{58}$ which is very popular for biased dynamics, is not available in VASP.

The thermodynamic integration exploits the formalism of Lagrange multipliers as implemented by T. Bucko in VASP. ${ }^{22}$ Constrained optimization have been performed using the 
same constraints as for the TI using the gadget interface. ${ }^{59}$ For the sake of computational efficiency, we did not attempt to optimize our structure on the free energy surface. ${ }^{60} \mathrm{As}$ detailed in the SI, the error estimates are based on the blocking algorithm that assesses the effect of correlation in the data on the statistical uncertainty ${ }^{61}$ and is implemented in the pyblock module ${ }^{62}$ For one point of the TI of $\mathrm{CO}$, we have repeated the simulation four times starting with different initial conditions. This lead to a free energy gradient of $-0.6 \mathrm{eV} / \AA$, with a standard deviation (between the four averages) of $1.5 \mathrm{eV} / \AA$, which compares well with the average estimated from the original simulations $(-0.9 \mathrm{eV} / \AA)$ and its $95 \%$ uncertainty interval $(1.3 \mathrm{eV} / \AA)$.

\section{Results and Discussion}

With the aim to determine various adsorption scenarios, we have investigated six different systems that cover strong and weak adsorptions, meta-stable chemisorbed states and adsorption energy profiles with several minima. The appearance of an adsorption barrier can, in general, have two origins. First, rotations that are not parallel to the surface are suppressed, which, depending on the temperature and interaction with the surface, can show up as a rotation-induced transition state (TS). Second, there might be a state crossing, inducing a chemical barrier. We have first focused on $\mathrm{CO}$ on $\mathrm{Ru}(0001)$ as it has previously been intensively studied experimentally and theoretically and shows a transient physisorbed state upon excitation with an optical laser, separated from the chemisorption minimum by a rotation-induced TS. We have then explored the desorption processes of $\mathrm{CO}_{2}$ on $\mathrm{Pt}(111)$, which is a typical case for state crossing upon adsorption. Together with formic acid, it is a species of interest for direct formic acid fuel cells or the hydrogenation of $\mathrm{CO}_{2} \cdot{ }^{63}$ We have finally considered three different aromatic molecules, namely benzene $\mathrm{C}_{6} \mathrm{H}_{6}$, phenol $\mathrm{C}_{6} \mathrm{H}_{5} \mathrm{OH}$ and hexachlorobenzene $\mathrm{C}_{6} \mathrm{Cl}_{6}$ on $\mathrm{Pt}(111)$. The first two are the simplest aromatic models of relevance in oil and biomass conversion, and $\mathrm{C}_{6} \mathrm{Cl}_{6}$ has the particularity of displaying three 
distinct adsorption states. For the aforementioned six systems, we have performed extensive AIMD simulations (100-150 ps per adsorbate) at the periodic DFT level. The results of the $\mathrm{TI}$ are compared with the energy profiles at $0 \mathrm{~K}$ and to the $0 \mathrm{~K}$ results in conjunction with three flavors of vertical thermal corrections (see SI).

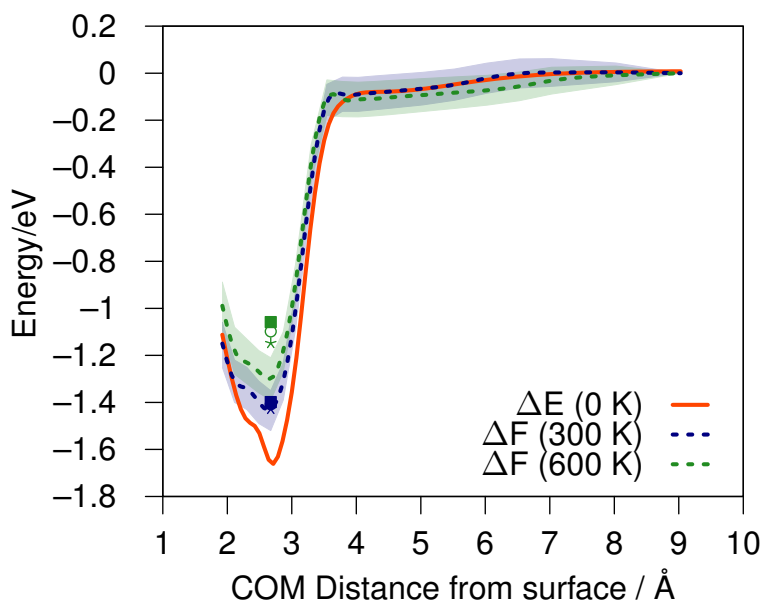

Figure 1: Adsorption energy (in red) and adsorption free energy at $300 \mathrm{~K}$ (blue) and $600 \mathrm{~K}$ (green) of $\mathrm{CO}$ on a $\mathrm{Ru}(0001)$ surface as a function of the center of mass (COM) to surface distance. As discussed in the text, the temperatures refer to the thermostat settings. Squares: RRHO; asterisks: Cut-off; open circles: Renormalization. The lines connect the evaluated points through a cubic spline. The shaded area corresponds to the $95 \%$ confidence interval of the thermodynamic integration.

Adsorption of carbon monoxide on the $\mathrm{Ru}(0001)$ surface was extensively studied by Pettersson and co-workers, who conducted computations to interpret experiments on the laser induced $\mathrm{CO}$ desorption dynamics that evidenced a physisorption minimum at high temperature in addition to the chemisorbed state. ${ }^{53,64}$ Applying a methodology put forward by Tully, ${ }^{65}$ these simulations were done quasi-analytically, using a parametrized molecule-surface interaction potential and integrating it according to Boltzmann statistics at finite temperature to obtain free energy profiles. These free energy profiles were referenced to the "desorption transition state", formally reached at infinite distance between the adsorbate and the surface. In this "desorption transition state", all translation and rotation degrees are recovered at the exception of the translation degree perpendicular to the surface which is the reaction coordinate. 
Here we use $a b$ initio MD, just like in all of the following examples, in combination with thermodynamic integration. Note that the actual average temperatures of $\mathrm{CO}$ are $\sim 230$ and $\sim 480 \mathrm{~K}$ instead of 300 and $600 \mathrm{~K}$. As shown in the Figure S1, such a deviation is to be expected for a diatomic species where effectively one degree of freedom is lost due to the constraint, necessary to perform the thermodynamic integration. Since the surface temperature is not affected by this loss of degree of freedom and thus is at the nominal temperature of the thermostat, we still refer to these simulations as corresponding to 300 and $600 \mathrm{~K}$, respectively. Increasing the temperature from $0 \mathrm{~K}$ to $300 \mathrm{~K}$ and then $600 \mathrm{~K}$, we observe the expected raise in free energy of the bottom of the well relative to this reference state which reflects the fact that adsorption is disfavored entropically by an increase in temperature.

In ref 53, the barrier due to suppressed rotations starts to be visible at around $600 \mathrm{~K}$. Figure 1 shows that the free energy profile at $300 \mathrm{~K}$ is still barrierless, while at $600 \mathrm{~K}$ a very small barrier $\sim 0.02 \mathrm{eV}$ becomes visible. Hence, our AIMD results are in qualitative agreement with the previous reports, where the barrier at $500 \mathrm{~K}$ was about $0.1 \mathrm{eV}$. The quantitative differences that could be observed by a detailed comparison are, apart to differences in the computational settings (e.g., the adsorption energy at $0 \mathrm{~K}$ is $\sim 1.4 \mathrm{eV}$ in ref 53 while we find it at $1.7 \mathrm{eV})$, due to two main effects. First, our $\mathrm{p}(2 \times 2)$ unit cell (taken from ref 53 ) is too small to prevent the interaction of the CO molecule with its periodic images. Second, our simulations take the thermal surface corrugation into account, which was not the case in ref 53. Furthermore, $\mathrm{CO}$ is allowed to diffuse between the hollow (preferred for distances smaller than $2.2 \AA$ according to our MDs) and the top site, which broadens the chemisorption minimum.

In summary, the $\mathrm{CO} @ \mathrm{Ru}(0001)$ adsorption example demonstrates good agreement with previous reports, validating the applied numerical settings and computational approach that can be applied to arbitrary systems.

Next, we turn to the comparison between carbon dioxide and formic acid adsorption on 
$\mathrm{Pt}(111)$, see Figure 2. Even though these two molecules contain the $\mathrm{O}-\mathrm{C}-\mathrm{O}$ backbone and are not strongly adsorbed on $\mathrm{Pt}(111)$, their adsorption energy profile is very different and characteristic for two different classes of compounds. Formic acid is only weakly adsorbed (0.4 $\mathrm{eV}$ at $0 \mathrm{~K}$ ) and its adsorption free energy gets halved at $300 \mathrm{~K}$. Carbon dioxide, on the other hand, shows an activated adsorption, with the chemisorption minimum being meta-stable, i.e., higher in energy than the desorbed state. The activation energy arises from a state crossing, between the linear $\mathrm{CO}_{2}$ molecule in the gas-phase and the bent adsorbate. The adsorption free energy barrier is increased by $0.15 \mathrm{eV}$ at $300 \mathrm{~K}$ temperature, which contrasts with the chemisorption minimum which is destabilized by twice this amount, thus reducing the desorption barrier from $\sim 0.3 \mathrm{eV}$ to only $\sim 0.15 \mathrm{eV}$.
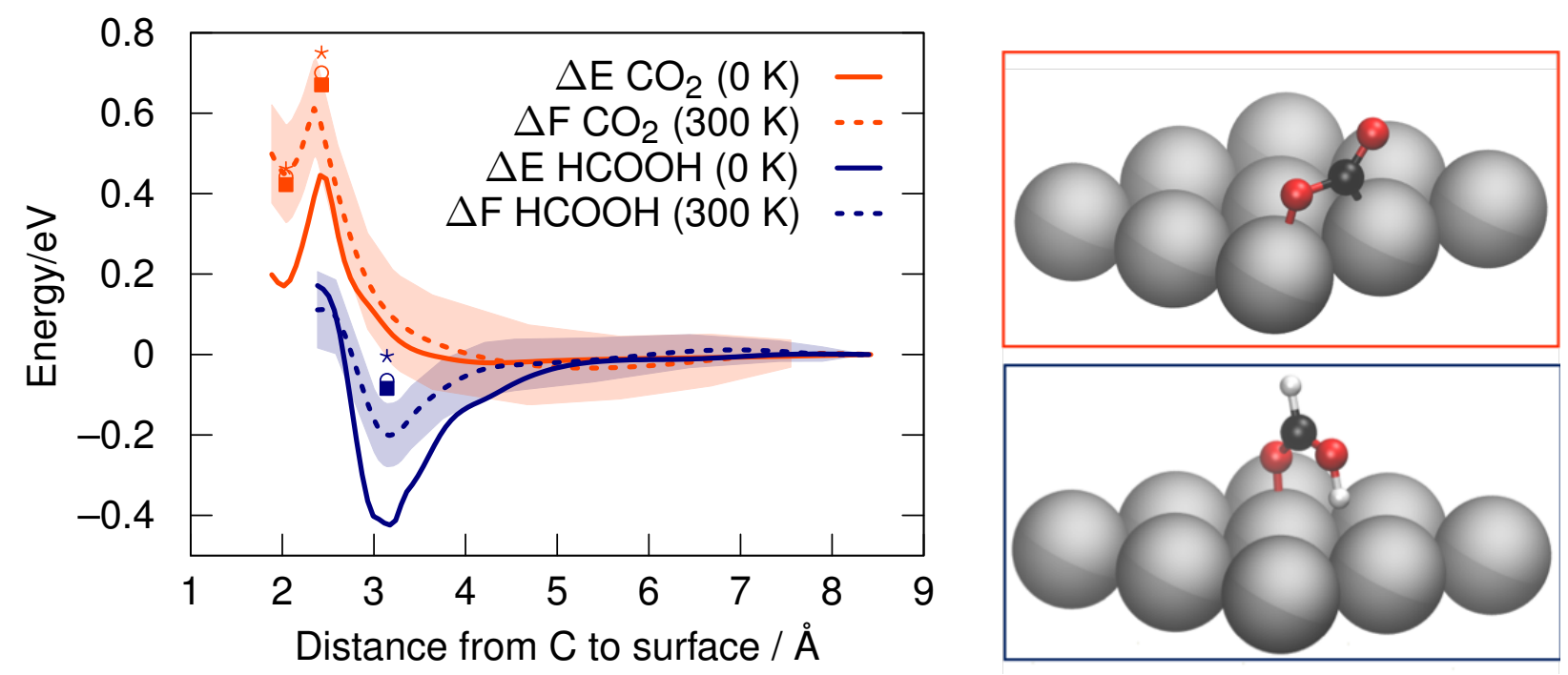

Figure 2: Left: Adsorption (free) energy ( $\Delta \mathrm{E}$ (full line) and $\Delta \mathrm{F}$ (300 $\mathrm{K}$, broken lines), respectively) of $\mathrm{CO}_{2}$ (red) and formic acid (blue) on a $\mathrm{Pt}(111)$ surface as a function of the carbon to surface distance. Squares: RRHO; asterisks: Cut-off; open circles: Renormalization. The shaded area corresponds to the $95 \%$ confidence interval of the thermodynamic integration. Right: Molecular structures of chemisorbed $\mathrm{CO}_{2}$ and physisorbed formic acid.

Having discussed these model systems, we apply the same methodology to the adsorption of aromatic molecules. These compounds are also key intermediates in the transformation of both the petroleum and biomass feedstocks. ${ }^{66}$ In Figure 3 we compare the adsorption energy and free energy of benzene, phenol and hexachlorobenzene $\mathrm{C}_{6} \mathrm{Cl}_{6}$ that happens to have a 

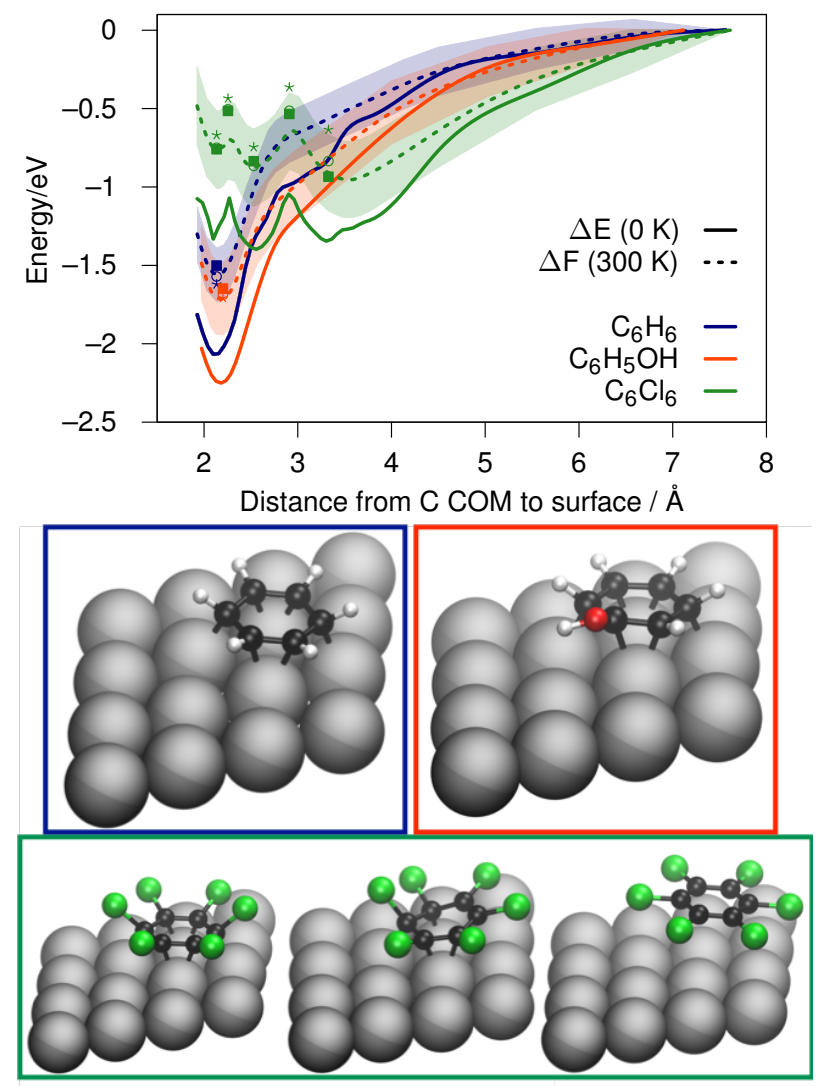

Figure 3: Top: Adsorption (free) energies ( $\Delta \mathrm{E}$ (full lines) and $\Delta \mathrm{F}$ (300 $\mathrm{K}$, dashed lines), respectively) for benzene (blue), phenol (red) and $\mathrm{C}_{6} \mathrm{Cl}_{6}$ (green) as a function of the $\mathrm{Pt}(111)$ surface - center of carbon atoms distance. Squares: RRHO; asterisks: Cut-off; open circles: Renormalization. The shaded area corresponds to the $95 \%$ confidence interval of the thermodynamic integration. Bottom: Geometries of chemisorbed benzene, phenol and the three minima (from left to right: $\eta^{6}, \eta^{3}$ and physisorption) of hexachlorobenzene. 
complex free energy landscape with three different adsorbed states.

Benzene shows the typical chemisorption minimum at $\sim 2.15 \AA$ above the surface, with its characteristic ring deformation. However, there is no physisorbed state in the $0 \mathrm{~K}$ profile, just a shoulder around $3 \AA$. This shoulder is less pronounced in the case of phenol, which moreover shows a deeper chemisorption minimum. This has recently been attributed to the $\mathrm{OH}$ substituent, that indirectly increases the affinity of the ring with the metal surface. ${ }^{2} \mathrm{C}_{6} \mathrm{Cl}_{6}$ is an analogue of benzene and could, therefore, be expected to have a very similar adsorption energy profile. However, this is far from being the case, as already reported earlier. ${ }^{67}$ At our level of theory, we find three adsorption energy minima: a physisorption minimum around 3.5 $\AA$ from the surface, an $\eta^{3}$ mode at $2.8 \AA$ and, finally, at a distance of $\sim 2.15 \AA$ the expected $\eta^{6}$ adsorption mode. Between each of these states, a transition state can be identified. The significantly different character of the various minima and the comparison with more "typical" aromatic systems (benzene and phenol) might reveal a different behavior with respect to the free energy corrections.

Starting with the case of benzene, we note that the temperature of $300 \mathrm{~K}$ preserves the qualitative features i.e., the shoulder observed at $0 \mathrm{~K}$ remains as a shoulder and does not become a stable state on the free energy surface. Similarly, the free energy profile of phenol does not qualitatively change at $300 \mathrm{~K}$ compared to $0 \mathrm{~K}$. Finally, the three minima of $\mathrm{C}_{6} \mathrm{Cl}_{6}$ remain distinct at $300 \mathrm{~K}$. The $\eta^{6}$ mode is more destabilized than the other minima and the small barrier towards the $\eta^{3}$ mode indicates that this adsorption mode is of little importance at room temperature. Alike, the $\eta^{3}$ mode is more destabilized than the physisorption minimum, so that the latter becomes the most stable state at room temperature. As a consequence, when considering the reactivity of $\mathrm{C}_{6} \mathrm{Cl}_{6}$ over $\mathrm{Pt}(111)$, Eley-Rideal type mechanisms cannot be discarded, and catalytic transformation could occur with the aromatic group never fully adsorbed on the surface. In summary, the relative impact of thermal corrections on these three cases (benzene, phenol, $\mathrm{C}_{6} \mathrm{Cl}_{6}$ ) corresponds to the chemical intuition. When already present on the potential energy surface (i.e., at $0 \mathrm{~K}$ ), the physisorption minimum becomes the 
most favored with an increase of temperature. Let us underline that the free energy correction to the $0 \mathrm{~K}$ result is, with $\sim 0.5 \mathrm{eV}$ or $20-30 \%$ of $\mathrm{E}_{\mathrm{ads}}(0 \mathrm{~K})$ near the minima, significant.

The final question to be addressed herein is the comparison with computationally cheap vertical thermal corrections for including free energy corrections. Following earlier works, ${ }^{21}$ we have to recognize that the direct comparison is necessarily skewed: AIMD is based on classical trajectories, neglecting the quantized nature of vibrations, which induces deviations at low temperatures.

In the vertical thermal corrections statistical mechanics is used to derive the free energy corrections that need to be applied to the $0 \mathrm{~K}$ electronic energies. As described in the computational details, we test the standard RRHO approach, but also a scheme based on the renormalization of low frequency modes to a fixed value ${ }^{54}$ or ignoring them altogether, which we call "cut-off". ${ }^{37}$ These three flavors are compared in Table S2 and the squares, circles and asterisks in Figures 1, 2 and 3 refer to RRHO, renormalization and cut-off, respectively. Overall, the results are quite close, with mean absolute deviations (MADs) compared to TI of $0.08 \mathrm{eV}$ (RRHO) to $0.15 \mathrm{eV}$ (cut-off) (see Table S2). Even just accounting for $-\mathrm{T} \Delta \mathrm{S}_{\text {trans }}$ (and thus neglecting the vibrational and rotational contributions) leads to a MAD of $0.12 \mathrm{eV}$. As is also evident from the figures, the statistical uncertainties (95\% confidence interval) of the $\mathrm{TI}$ are also non-negligible $(0.18 \mathrm{eV}$ for maxima/minima on average), especially for the aromatic molecules, where the integration points at "long" distance are spaced by $1 \AA$, which leads to a rapid increase in the uncertainty.

For the minima, the agreement between RRHO and TI is acceptably good, so that we recommend using this approach in general at least at temperatures close to room-temperature. At higher temperatures, the renormalization approach is more robust in the sense that the low frequency modes do not lead to excessively high entropic contributions, while the results at $300 \mathrm{~K}$ barely deviate from RRHO, a deviation that can be further lowered by using a renormalization value to 50 instead of $100 \mathrm{~cm}^{-1}$.

Despite this, the vertical corrections obviously can neither predict the appearance of a 
physisorption minimum, nor the change in the position of the physisorption minimum, which can get shifted to longer distances at higher temperatures $(+0.2 \AA)$, as can be observed for hexachlorobenzene in Figure 3. The agreement between RRHO and TI is worse for transition states, where the vertical corrections lead to higher barriers than the TI in both studied systems, i.e., $\mathrm{CO}_{2}$ and $\mathrm{C}_{6} \mathrm{Cl}_{6}$. To get further insight, we monitored the main distances and angles of $\mathrm{CO}_{2}$ along the desorption process. As shown in Figures S2-S4, the distributions of typical parameters such as the shortest $\mathrm{Pt}-\mathrm{C}$ distance and the $\mathrm{O}-\mathrm{C}-\mathrm{O}$ angle, are very sensitive to the precise value of the reaction coordinate. Hence, only very dense sampling around the transition state allows an accurate estimate of both, the transition state structure and energetics. We thus suggest that the lowering of barriers obtained by TI compared to RRHO is an indication of the importance of thermal fluctuations of the metal surface and the associated anharmonic response of the transition state for driving reaction barriers down.

This is in agreement with molecular beam experiments ${ }^{68,69}$ and the corresponding simulations ${ }^{69,70}$ which have reported that the thermal surface deformation lowers the activation energy for $\mathrm{C}-\mathrm{H}$ bond breaking over $\mathrm{Pd}(111)$ and $\mathrm{Ni}(111)$. All in all, energy barriers are much more sensitive to the free energy approximations than reaction energies and can be lower at finite temperatures than estimated from statistical mechanics, but care has to be take to properly converge them in the numerical simulations.

\section{Conclusion}

We have compared first principles based thermodynamic integration to three approximations for vertical, statistical mechanical thermal corrections of six typical adsorbates $\left(\mathrm{CO}, \mathrm{CO}_{2}\right.$, formic acid, benzene, phenol and hexachlorobenzene) on metallic surfaces. Our results suggest that the standard harmonic oscillator, the use of a cut-off and the renormalization of low frequency modes all give results in good agreement with TI for the chemisorption minima, so that the standard RRHO approach can be recommended. The agreement is, however, 
0.1-0.2 eV worse for transition states. Hence, even at $300 \mathrm{~K}$ the effect of the thermal surface roughness and associated anharmonicity for the transition state cannot be neglected when high accuracy is sought for.

\section{Acknowledgement}

The authors thank the SYSPROD project and AXELERA Pôle de Compétitivité for financial support (PSMN Data Center). This work was granted access to the HPC resources of CINES and IDRIS under the allocation 2014-080609 made by GENCI. This work benefited from the support of the project MuSiC ANR-14-CE06-0030 of the French National Research Agency (ANR). Work at UCLA was supported by the U.S. Department of Energy (DOE), Office of Science, Office of Basic Energy Sciences (BES), Materials Sciences and Engineering Division under Award \# DE-SC0012573. We are grateful to T. Bucko for providing the Gadget interface.

\section{Supporting Information Available}

The supporting information contains the geometries of the stationary points, discussion of the thermodynamics at the surface/gas interface and the need to reference the free energy profiles to the transition state of desorption and additional figures and tables concerning the details of the thermal corrections, ZPE and the thermostat. An excel sheet with the raw data for the thermodynamic integration and uncertainty propagation is also provided. This material is available free of charge via the Internet at http://pubs.acs.org/.

\section{References}

(1) Yang, B.; Burch, R.; Hardacre, C.; Headdock, G.; Hu, P. Understanding the Optimal Adsorption Energies for Catalyst Screening in Heterogeneous Catalysis. ACS Catal. 
2014, 4, 182-186.

(2) Réocreux, R.; Huynh, M.; Michel, C.; Sautet, P. Controlling the Adsorption of Aromatic Compounds on Pt(111) with Oxygenate Substituents: From DFT to Simple Molecular Descriptors. J. Phys. Chem. Lett. 2016, 7, 2074-2079.

(3) Loffreda, D.; Delbecq, F.; Vigné, F.; Sautet, P. Catalytic Hydrogenation of Unsaturated Aldehydes on Pt(111): Understanding the Selectivity from First-Principles Calculations. Angew. Chem., Int. Ed. 2005, 44, 5279-5282.

(4) Viola, A.; Peron, J.; Kazmierczak, K.; Giraud, M.; Michel, C.; Sicard, L.; Perret, N.; Beaunier, P.; Sicard, M.; Besson, M. et al. Unsupported Shaped Cobalt Nanoparticles as Efficient and Recyclable Catalysts for the Solvent-Free Acceptorless Dehydrogenation of Alcohols. Catalysis Science \& Technology 2018, 8, 562-572.

(5) Mom, R. V.; Melissen, S. T. A. G.; Sautet, P.; Frenken, J. W. M.; Steinmann, S. N.; Groot, I. M. N. The Pressure Gap for Thiols: Methanethiol Self-Assembly on Au(111) from Vacuum to 1 bar. J. Phys. Chem. C 2019, 123, 12382-12389.

(6) Greeley, J.; Norskov, J. K.; Mavrikakis, M. Electronic Structure and Catalysis on Metal Surfaces. Annu. Rev. Phys. Chem. 2002, 53, 319-348.

(7) van Santen, R. A.; Sautet, P. Computational Methods in Catalysis and Materials Science: An Introduction for Scientists and Engineers; Wiley-VCH Verlag GmbH \& Co. KGaA: Weinheim, 2009.

(8) Klimes, J.; Bowler, D. R.; Michaelides, A. Chemical Accuracy for the Van der Waals Density Functional. J. Phys.: Condens. Matter 2010, 22, 022201.

(9) Wellendorff, J.; Lundgaard, K. T.; Møgelhøj, A.; Petzold, V.; Landis, D. D.; Nørskov, J. K.; Bligaard, T.; Jacobsen, K. W. Density Functionals for Surface Sci- 
ence: Exchange-Correlation Model Development With Bayesian Error Estimation. Phys. Rev. B 2012, 85, 235149.

(10) Gautier, S.; Steinmann, S. N.; Michel, C.; Fleurat-Lessard, P.; Sautet, P. Molecular Adsorption at Pt(111). How Accurate are DFT Functionals? Phys. Chem. Chem. Phys. 2015, 17, 28921-28930.

(11) Duanmu, K.; Truhlar, D. G. Validation of Density Functionals for Adsorption Energies on Transition Metal Surfaces. J. Chem. Theory Comput. 2017, 13, 835-842.

(12) Hensley, A. J. R.; Ghale, K.; Rieg, C.; Dang, T.; Anderst, E.; Studt, F.; Campbell, C. T.; McEwen, J.-S.; Xu, Y. DFT-Based Method for More Accurate Adsorption Energies: An Adaptive Sum of Energies from RPBE and vdW Density Functionals. J. Phys. Chem. C 2017, 121, 4937-4945.

(13) Borroni-Bird, C. E.; Al-Sarraf, N.; Andersoon, S.; King, D. A. Single Crystal Adsorption Microcalorimetry. Chem. Phys. Lett. 1991, 183, 516-520.

(14) Stuckless, J. T.; Frei, N. A.; Campbell, C. T. A Novel Single-Crystal Adsorption Calorimeter and Additions for Determining Metal Adsorption and Adhesion Energies. Rev. Sci. Instrum. 1998, 69, 2427-2438.

(15) Campbell, C. T.; Sellers, J. R. V. The Entropies of Adsorbed Molecules. J. Am. Chem. Soc. 2012, 134, 18109-18115.

(16) Redhead, P. Thermal Desorption of Gases. Vacuum 1962, 12, 203-211.

(17) Réocreux, R.; Ould Hamou, C. A.; Michel, C.; Giorgi, J. B.; Sautet, P. Decomposition Mechanism of Anisole on Pt(111): Combining Single-Crystal Experiments and FirstPrinciples Calculations. ACS Catal. 2016, 6, 8166-8178.

(18) Flaherty, D. W.; Iglesia, E. Transition-State Enthalpy and Entropy Effects on Reactivity 
and Selectivity in Hydrogenolysis of n-Alkanes. J. Am. Chem. Soc. 2013, 135, 1858618599.

(19) Jorgensen, M.; Gronbeck, H. Adsorbate Entropies with Complete Potential Energy Sampling in Microkinetic Modeling. J. Phys. Chem. C 2017, 121, 7199-7207.

(20) Bajpai, A.; Mehta, P.; Frey, K.; Lehmer, A. M.; Schneider, W. F. Benchmark FirstPrinciples Calculations of Adsorbate Free Energies. ACS Catal. 2018, 8, 1945-1954.

(21) Kelly, E.; Seth, M.; Ziegler, T. Calculation of Free Energy Profiles for Elementary Bimolecular Reactions by ab Initio Molecular Dynamics: Sampling Methods and Thermostat Considerations. J. Phys. Chem. A 2004, 108, 2167-2180.

(22) Bucko, T. Ab Initio Calculations of Free-Energy Reaction Barriers. J. Phys.: Condens. Matter 2008, 20, 064211.

(23) Hoefling, M.; Iori, F.; Corni, S.; Gottschalk, K.-E. Interaction of Amino Acids with the $\mathrm{Au}(111)$ Surface: Adsorption Free Energies from Molecular Dynamics Simulations. Langmuir 2010, 26, 8347-8351.

(24) Kerisit, S.; Parker, S. C. Free Energy of Adsorption of Water and Metal Ions on the 1014 Calcite Surface. J. Am. Chem. Soc. 2004, 126, 10152-10161.

(25) Suter, J. L.; Boek, E. S.; Sprik, M. Adsorption of a Sodium Ion on a Smectite Clay from Constrained Ab Initio Molecular Dynamics Simulations. J. Phys. Chem. C 2008, 112, $18832-18839$.

(26) Brandt, E. G.; Lyubartsev, A. P. Molecular Dynamics Simulations of Adsorption of Amino Acid Side Chain Analogues and a Titanium Binding Peptide on the TiO2 (100) Surface. J. Phys. Chem. C 2015, 119, 18126-18139.

(27) YazdanYar, A.; Aschauer, U.; Bowen, P. Adsorption Free Energy of Single Amino Acids 
at the Rutile (110)/Water Interface Studied by Well-Tempered Metadynamics. J. Phys. Chem. C 2018, 122, 11355-11363.

(28) Truhlar, D. G.; Garrett, B. C.; Klippenstein, S. J. Current Status of Transition-State Theory. J. Phys. Chem. 1996, 100, 12771.

(29) Peter, C.; Oostenbrink, C.; van Dorp, A.; van Gunsteren, W. F. Estimating Entropies from Molecular Dynamics Simulations. J. Chem. Phys. 2004, 120, 2652-2661.

(30) Lin, S.-T.; Maiti, P. K.; Goddard, W. A. Two-Phase Thermodynamic Model for Efficient and Accurate Absolute Entropy of Water from Molecular Dynamics Simulations. J. Phys. Chem. B 2010, 114, 8191-8198.

(31) Pitt, I. G.; Gilbert, R. G.; Ryan, K. R. Application of Transition-State Theory to Gas-Surface Reactions: Barrierless Adsorption on Clean Surfaces. J. Phys. Chem. 1994, 98, 13001-13010.

(32) Campbell, C. T.; Arnadottir, L.; Sellers, J. R. V. Kinetic Prefactors of Reactions on Solid Surfaces. Z. Phys. Chem. 2013, 227, 1435-1454.

(33) Campbell, C. T.; Sprowl, L. H.; Arnadottir, L. Equilibrium Constants and Rate Constants for Adsorbates: Two-Dimensional (2D) Ideal Gas, 2D Ideal Lattice Gas, and Ideal Hindered Translator Models. J. Phys. Chem. C 2016, 120, 10283-10297.

(34) Piccini, G.; Sauer, J. Quantum Chemical Free Energies: Structure Optimization and Vibrational Frequencies in Normal Modes. J. Chem. Theory Comput. 2013, 9, 50385045 .

(35) Piccini, G.; Sauer, J. Effect of Anharmonicity on Adsorption Thermodynamics. J. Chem. Theory Comput. 2014, 10, 2479-2487.

(36) Grimme, S. Supramolecular Binding Thermodynamics by Dispersion-Corrected Density Functional Theory. Chem. Eur. J. 2012, 18, 9955-9964. 
(37) Steinmann, S. N.; Michel, C.; Schwiedernoch, R.; Filhol, J.-S.; Sautet, P. Modeling the $\mathrm{HCOOH} / \mathrm{CO}_{2}$ Electrocatalytic Reaction: When Details Are Key. ChemPhysChem 2015, 16, 2307-2311.

(38) Cnudde, P.; De Wispelaere, K.; Van der Mynsbrugge, J.; Waroquier, M.; Van Speybroeck, V. Effect of Temperature and Branching on the Nature and Stability of Alkene Cracking Intermediates in H-ZSM-5. J. Catal. 2017, 345, 53-69.

(39) Rey, J.; Gomez, A.; Raybaud, P.; Chizallet, C.; Bučko, T. On the Origin of the Difference Between Type A and Type B Skeletal Isomerization of Alkenes Catalyzed by Zeolites: The Crucial Input of Ab Initio Molecular Dynamics. J. Catal. 2019, 373, 361-373.

(40) Li, Y.-P.; Gomes, J.; Mallikarjun Sharada, S.; Bell, A. T.; Head-Gordon, M. Improved Force-Field Parameters for QM/MM Simulations of the Energies of Adsorption for Molecules in Zeolites and a Free Rotor Correction to the Rigid Rotor Harmonic Oscillator Model for Adsorption Enthalpies. J. Phys. Chem. C 2015, 119, 1840-1850.

(41) Bucko, T.; Benco, L.; Hafner, J.; Angyan, J. G. Proton Exchange of Small Hydrocarbons Over Acidic Chabazite: Ab Initio Study of Entropic Effects. J. Catal. 2007, 250, $171-183$.

(42) Jiang, T.; Göltl, F.; Bulo, R. E.; Sautet, P. Effect of Temperature on the Adsorption of Short Alkanes in the Zeolite SSZ-13-Adapting Adsorption Isotherms to Microporous Materials. ACS Catal. 2014, 4, 2351-2358.

(43) Li, H.; Paolucci, C.; Schneider, W. F. Zeolite Adsorption Free Energies from Ab Initio Potentials of Mean Force. J. Chem. Theory Comput. 2018, 14, 929-938.

(44) Gilson, M. K.; Given, J. A.; Bush, B. L.; McCammon, J. A. The StatisticalThermodynamic Basis for Computation of Binding Affinities: A Critical Review. Biophys. J. 1997, 72, 1047-1069. 
(45) Carlsson, J.; Aqvist, J. Absolute and Relative Entropies from Computer Simulation with Applications to Ligand Binding. J. Phys. Chem. B 2005, 109, 6448-6456.

(46) Besora, M.; Vidossich, P.; Lledos, A.; Ujaque, G.; Maseras, F. Calculation of Reaction Free Energies in Solution: A Comparison of Current Approaches. J. Phys. Chem. A 2018, 122, 1392-1399.

(47) Zhou, H.-X.; Gilson, M. K. Theory of Free Energy and Entropy in Noncovalent Binding. Chem. Rev. 2009, 109, 4092-4107.

(48) Kresse, G.; Hafner, J. Ab Initio Molecular Dynamics for Liquid Metals. Phys. Rev. B 1993, 47, 558.

(49) Kresse, G.; Furthmuller, J. Efficient Iterative Schemes for Ab Initio Total-Energy Calculations Using a Plane-Wave Basis set. Phys. Rev. B 1996, 54, 11169.

(50) Blochl, P. E. Projector Augmented-Wave Method. Phys. Rev. B 1994, 50, 17953.

(51) Kresse, G.; Joubert, D. From Ultrasoft Pseudopotentials to the Projector AugmentedWave Method. Phys. Rev. B 1999, 59, 1758.

(52) Perdew, J. P.; Burke, K.; Ernzerhof, M. Generalized Gradient Approximation Made Simple. Phys. Rev. Lett. 1996, 77, 3865.

(53) Dell'Angela, M.; Anniyev, T.; Beye, M.; Coffee, R.; Fohlisch, A.; Gladh, J.; Katayama, T.; Kaya, S.; Krupin, O.; LaRue, J. et al. Real-Time Observation of Surface Bond Breaking with an X-ray Laser. Science 2013, 339, 1302-1305.

(54) Zhao, Y.; Truhlar, D. G. Computational Characterization and Modeling of Buckyball Tweezers: Density Functional Study of Concave-Convert $\pi \cdots \pi$ Interactions. Phys. Chem. Chem. Phys. 2008, 10, 2813-2818. 
(55) Pascale, F.; Tosoni, S.; Zicovich-Wilson, C.; Ugliengo, P.; Orlando, R.; Dovesi, R. Vibrational Spectrum of Brucite, $\mathrm{Mg}(\mathrm{OH})_{2}$ : A Periodic Ab Initio Quantum Mechanical Calculation Including OH Anharmonicity. Chem. Phys. Lett. 2004, 396, 308-315.

(56) Andersen, H. C. Molecular Dynamics Simulations at Constant Pressure and/or Temperature. J. Chem. Phys. 1980, 72, 2384-2393.

(57) Koopman, E. A.; Lowe, C. P. Advantages of a Lowe-Andersen Thermostat in Molecular Dynamics Simulations. J. Chem. Phys. 2006, 124, 204103.

(58) Bussi, G.; Donadio, D.; Parrinello, M. Canonical Sampling Through Velocity Rescaling. J. Chem. Phys. 2007, 126, 014101.

(59) Bucko, T.; Hafner, J.; Angyan, J. G. Geometry Optimization of Periodic Systems Using Internal Coordinates. J. Chem. Phys. 2005, 122, 124508.

(60) Fleurat-Lessard, P.; Ziegler, T. Tracing the Minimum-Energy Path on the Free-Energy Surface. J. Chem. Phys. 2005, 123, 084101.

(61) Flyvbjerg, H.; Petersen, H. G. Error Estimates on Averages of Correlated Data. J. Chem. Phys. 1989, 91, 461-466.

(62) Spencer, J. Pyblock, see https://github.com/jsspencer/pyblock/blob/master/ docs/index.rst. 2014.

(63) Whipple, D. T.; Kenis, P. J. A. Prospects of $\mathrm{CO}_{2}$ Utilization via Direct Heterogeneous Electrochemical Reduction. J. Phys. Chem. Lett. 2010, 1, 3451-3458.

(64) Xin, H.; LaRue, J.; Oberg, H.; Beye, M.; Dell'Angela, M.; Turner, J.; Gladh, J.; Ng, M.; Sellberg, J.; Kaya, S. et al. Strong Influence of Coadsorbate Interaction on CO Desorption Dynamics on $\mathrm{Ru}(0001)$ Probed by Ultrafast X-Ray Spectroscopy and Ab Initio Simulations. Phys. Rev. Lett. 2015, 114, 156101. 
(65) Doren, D. J.; Tully, J. C. Precursor-Mediated Adsorption and Desorption: A Theoretical Analysis. Langmuir 1988, 4, 256-268.

(66) Réocreux, R.; Michel, C. Rational Design of Heterogeneous Catalysts for Biomass Conversion - Inputs from Computational Chemistry. Curr. Opin. Green Sustain. Chem. 2018, 10, 51-59.

(67) Pekoz, R.; Johnston, K.; Donadio, D. Tuning the Adsorption of Aromatic Molecules on Platinum via Halogenation. J. Phys. Chem. C 2014, 118, 6235-6241.

(68) Harris, J.; Simon, J.; Luntz, A. C.; Mullins, C. B.; Rettner, C. T. Thermally Assisted Tunneling: $\mathrm{CH}_{4}$ Dissociation on Pt(111). Phys. Rev. Lett. 1991, 67, 652-655.

(69) Killelea, D. R.; Campbell, V. L.; Shuman, N. S.; Smith, R. R.; Utz, A. L. Surface Temperature Dependence of Methane Activation on Ni(111). J. Phys. Chem. C 2009, 113, 20618-20622.

(70) Lozano, A.; Shen, X. J.; Moiraghi, R.; Dong, W.; Busnengo, H. F. Cutting a Chemical Bond with Demon's Scissors: Mode- and Bond-Selective Reactivity of Methane on Metal Surfaces. Surf. Sci. 2015, 640, 25-35. 


\section{Graphical TOC Entry}

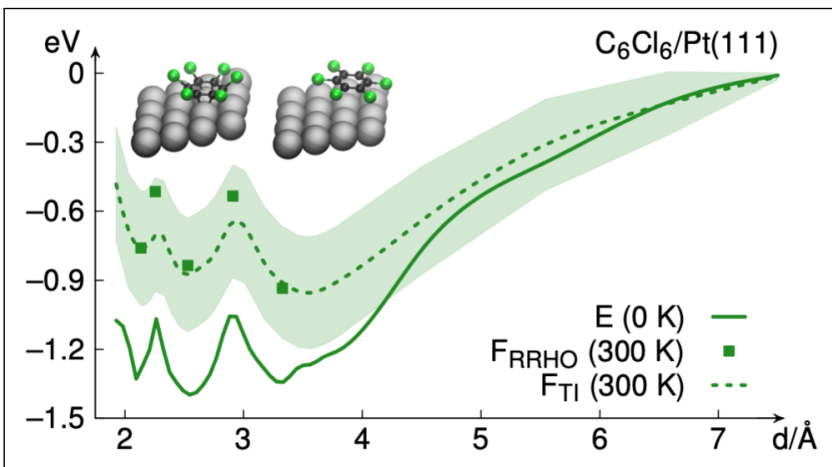

